\title{
General convergence analysis of stochastic first order methods for composite optimization
}

\section{Ion Necoara}

\begin{abstract}
In this paper we consider stochastic composite convex optimization problems with the objective function satisfying a stochastic bounded gradient condition, with or without a quadratic functional growth property. These models include the most well-known classes of objective functions analyzed in the literature: non-smooth Lipschitz functions and composition of a (potentially) non-smooth function and a smooth function, with or without strong convexity. Based on the flexibility offered by our optimization model we consider several variants of stochastic first order methods, such as the stochastic proximal gradient and the stochastic proximal point algorithms. Usually, the convergence theory for these methods has been derived for simple stochastic optimization models satisfying restrictive assumptions, the rates are in general sublinear and hold only for specific decreasing stepsizes. Hence, we analyze the convergence rates of stochastic first order methods with constant or variable stepsize under general assumptions covering a large class of objective functions. For constant stepsize we show that these methods can achieve linear convergence rate up to a constant proportional to the stepsize and under some strong stochastic bounded gradient condition even pure linear convergence. Moreover, when a variable stepsize is chosen we derive sublinear convergence rates for these stochastic first order methods. Finally, the stochastic gradient mapping and the Moreau smoothing mapping introduced in the present paper lead to simple and intuitive proofs.
\end{abstract}

Keywords Stochastic composite convex optimization · stochastic bounded gradient - quadratic functional growth · stochastic first order algorithms . convergence rates.

Mathematics Subject Classification (2000) 65K05 - 90C15 - 52A20.

Ion Necoara

Automatic Control and Systems Engineering Department, University Politehnica Bucharest, 060042 Bucharest, Romania, ion.necoara@acse.pub.ro 


\section{Introduction}

The randomness in most of the practical optimization applications led the stochastic optimization field to become an essential tool for many applied mathematics areas, such as machine learning and statistics 7, distributed control and signal processing [10], sensor networks [2] and others. In particular, in statistics and machine learning applications the optimization algorithms involve numerical computation of parameters for a system designed to make decisions based on yet unseen data. The recent success of certain optimization methods for statistics and machine learning problems has motivated increasingly great efforts into developments of new numerical algorithms or into analyzing deeper the existing ones.

In this paper we analyze a popular class of algorithms for solving stochastic composite convex optimization problems, that is stochastic first order (SFO) methods [1, 5, 7, 11, 12, 14, 19. We assume that we have access to an unbiased estimate of the gradient or of the proximal operator of a certain function we wish to minimize, which is key to scale up optimization and to address streaming settings where data arrive in time. In these scenarios, SFO methods independently sample an unbiased estimate of the gradient or of the proximal operator and then take a step along this direction with a certain stepsize length. These algorithms are typically the methods of choice in practice for many applications due to their cheap iteration and superior empirical performance. However, the theoretical convergence rates from the literature are usually sublinear and hold only for decreasing stepsizes. Moreover, the convergence theory treats separately smooth or non-smooth objective functions and covers usually unconstrained optimization models. On the other hand, in this paper we present a general framework for the analysis of SFO algorithms for solving general composite optimization problems, expressed in terms of expectation operator. This framework is based on the assumptions that the objective function satisfies a stochastic bounded gradient condition, with or without a quadratic functional growth property. These conditions include the most well-known classes of objective functions analyzed in the literature: nonsmooth Lipschitz functions and composition of a (potentially) non-smooth function and a smooth function, with or without strong convexity. Based on this framework we derive a complete convergence analysis for these SFO methods, that is stochastic proximal gradient and proximal point algorithms.

More specifically, a very popular approach for solving stochastic optimization problems, where the regularization function is the indicator function of some simple convex set, is the stochastic gradient descent (SGD) algorithm [5, 11, 12, 19. In this paper we complement and extend the previous results for SGD to a wider class of stochastic composite optimization problems having more general assumptions and to more general stochastic first order methods. In particular, we extend the convergence analysis of this well-known method to composite optimization problems having a smooth or non-smooth term and a general regularization term that leads to the stochastic proximal gradient (SPG) algorithm. Other results related to the convergence behavior 
of SPG can be found in [1,6,17. Furthermore, despite the fact that the computational performance of SPG may be good under certain circumstances, there is recent evidence of its instability for unappropriate parameters choice [7]. To avoid this behavior of SPG scheme, we also analyze the convergence behavior of stochastic proximal point (SPP) algorithm. Papers on SPP related to our work are e.g. [14, 18,20]. We show that these two methods, SPG and SPP, can achieve linear convergence rate up to a constant proportional to the stepsize and under some strong stochastic bounded gradient condition even pure linear convergence. We also prove that the strong stochastic bounded gradient condition is not only sufficient but also necessary for obtaining linear convergence. When the strong stochastic bounded gradient condition does not hold we show that restarted variants of these methods can still achieve linear convergence. Moreover, when variable stepsize is chosen we derive sublinear convergence rates for these stochastic first order methods. Finally, the stochastic gradient mapping and the Moreau smoothing mapping from this present paper lead to more elegant and intuitive proofs.

Content: This section continues with the presentation of our problem of interest and the main assumptions. In Section 2 we propose two stochastic first order methods and derive their convergence rates. Finally, Section 3 presents a restarted variant of these methods and analyze its convergence.

\subsection{Problem formulation}

Let $f, g: \mathbb{R}^{m} \times \Omega \rightarrow \overline{\mathbb{R}}$ be two proper convex functions in the first argument, where $\Omega$ is endowed with a probability distribution $\mathbf{P}$. Then, we consider the following general composite stochastic optimization problem:

$$
F^{*}=\min _{x \in \mathbb{R}^{m}} F(x) \quad(\stackrel{\text { def }}{=} f(x)+g(x)),
$$

where the two functions have stochastic representations in the form of expectation, i.e. $f(x)=\mathbf{E}[f(x, \xi)]$ and $g(x)=\mathbf{E}[g(x, \xi)]$. We assume that the expectation taken with respect to the random variable $\xi \in \Omega$ is finite for all $x \in \operatorname{dom} F$. Hence we consider a flexible splitting of our stochastic objective function $F(x)=\mathbf{E}[F(x, \xi)]$ in composite form as a sum of two terms:

$$
F(x, \xi)=f(x, \xi)+g(x, \xi) .
$$

In this paper we assume $f(\cdot, \xi)$ either differentiable or nondifferentiable function and we use, with some abuse of notation, the same notation for the gradient or subgradient of $f(\cdot, \xi)$ at $x$, that is $\nabla f(x, \xi) \in \partial f(x, \xi)$, where $\partial f(x, \xi)$ is either a singleton or a nonempty convex set. The other term, $g(x, \xi)$, is assumed to be simple and its structure known. We usually refer to $g$ as the regularizer and use the same notation as before $\nabla g(x, \xi) \in \partial g(x, \xi)$ for a (sub)gradient of $g(x, \xi)$ at $x$. We also assume in the sequel that to have access to either an unbiased stochastic estimates of the (sub)gradients of function $f$, i.e.:

$$
\mathbb{E}[\nabla f(x, \xi)] \in \partial f(x), \text { where } \nabla f(x, \xi) \in \partial f(x, \xi),
$$


or to stochastic estimates of the proximal operator of $f$, i.e.:

$$
\operatorname{prox}_{\gamma f(\cdot, \xi)}(x)=\arg \min _{y \in \mathbb{R}^{m}} f(y, \xi)+\frac{1}{2 \gamma}\|y-x\|^{2} .
$$

We refer to $\operatorname{prox}_{\gamma f(\cdot, \xi)}(x)$ as the proximal operator of the function $f(\cdot, \xi)$ at $x$ with stepsize $\gamma$. We always assume to have access to stochastic estimates of the proximal operator of $g$, i.e. $\operatorname{prox}_{\gamma g(\cdot, \xi)}(x)$. These are key assumptions in our stochastic optimization to scale up the numerical algorithms. Template (11) covers many applications in machine learning, statistics, signal processing, control and other areas, by appropriately choosing the functions $f$ and $g$.

1.2 Main assumptions

Let us denote by $X^{*}$ the optimal set of the convex problem (11) and for any $x \in \mathbb{R}^{m}$ we denote its projection onto $X^{*}$ by $\bar{x}$, that is $\bar{x}=\Pi_{X^{*}}(x)$. In this paper we consider additionally the following assumptions:

Assumption 1.1 The stochastic (sub)gradients of F satisfy a stochastic bounded gradient condition restricted on any segment $[x, \bar{x}]$, that is there exist nonnegative constants $L \geq 0$ and $B \geq 0$ such that:

$$
B^{2}+L(F(x)-F(\bar{x})) \geq \mathbf{E}_{\xi}\left[\|\nabla F(x, \xi)\|^{2}\right] \quad \forall x \in \operatorname{dom} F .
$$

Assumption 1.2 The function $F$ satisfies a quadratic functional growth condition restricted on any segment $[x, \bar{x}]$, that is there exists $\mu \geq 0$ such that:

$$
F(x)-F(\bar{x}) \geq \frac{\mu}{2}\|x-\bar{x}\|^{2} \quad \forall x \in \operatorname{dom} F .
$$

Note that none of the conditions (2) and/or (3) implies convexity of the function $F$. When $B=0$ we refer to (2) as the strong stochastic bounded gradient condition. Note that the strong stochastic bounded gradient condition has been considered in [9] for proving linear convergence of stochastic gradient descent method for solving convex feasibility problems. In this paper we generalize the strong stochastic bounded gradient condition from [9] by adding a positive constant $B$ in order to cover the most well-known classes of objective functions analyzed in the literature: non-smooth Lipschitz functions, and composition of a (potentially) non-smooth function and a smooth function. A similar stochastic bounded gradient condition of the form (2) has been also considered in 4 . in the context of non-smooth optimization. Further, the quadratic functional growth condition (3) has been considered in [8] for deterministic optimization, where it was proved that the class of smooth functions satisfying (3) is the largest one for which gradient method is converging linearly. We now present several classes of functions satisfying Assumptions 1.1 and 1.2. First note that Assumption 1.1 is very general and covers a large class of functionals, such as Lipschitz functions or functions having Lipschitz continuous gradients.

Example 1 [Non-smooth (Lipschitz) functions satisfy Assumption 1.1]: Assume that the functions $f(\cdot, \xi)$ and $g(\cdot, \xi)$ have bounded (sub)gradients:

$$
\|\nabla f(x, \xi)\| \leq B_{f} \quad \text { and } \quad\|\nabla g(x, \xi)\| \leq B_{g} \quad \forall x \in \operatorname{dom} F .
$$


Then, obviously Assumption 1.1 holds with $L=0 \quad$ and $\quad B^{2}=2 B_{f}^{2}+2 B_{g}^{2}$.

Example 2 [Smooth (Lipschitz gradient) functions satisfy Assumption 1.1]: Condition (2) contains the class of functions formed as a sum of two terms, one having Lipschitz continuous gradient and the other having bounded subgradients. Indeed, let us assume that $f(\cdot, \xi)$ has Lipschitz continuous gradient, i.e. there exists $L(\xi)>0$ such that:

$$
\|\nabla f(x, \xi)-\nabla f(\bar{x}, \xi)\| \leq L(\xi)\|x-\bar{x}\| \quad \forall x \in \operatorname{dom} F .
$$

Then, using standard arguments we have [13]:

$$
f(x, \xi)-f(\bar{x}, \xi) \geq\langle\nabla f(\bar{x}, \xi), x-\bar{x}\rangle+\frac{1}{2 L(\xi)}\|\nabla f(x, \xi)-\nabla f(\bar{x}, \xi)\|^{2} .
$$

Assuming that $L(\xi) \leq L_{f}$ for all $\xi \in \Omega$ and $g(\cdot, \xi)$ convex, then adding $g(x, \xi)-$ $g(\bar{x}, \xi) \geq\langle\nabla g(\bar{x}, \xi), x-\bar{x}\rangle$ in the previous inequality, where $\nabla g(\bar{x}, \xi) \in \partial g(\bar{x}, \xi)$, and then taking expectation w.r.t. $\xi$, we get:

$$
F(x)-F(\bar{x}) \geq\langle\nabla F(\bar{x}), x-\bar{x}\rangle+\frac{1}{2 L_{f}} \mathbf{E}\left[\|\nabla f(x, \xi)-\nabla f(\bar{x}, \xi)\|^{2}\right],
$$

where we used that $\nabla f(\bar{x}, \xi)$ and $\nabla g(\bar{x}, \xi)$ are unbiased stochastic estimates of the (sub)gradients of $f$ and $g$ and thus $\nabla F(\bar{x})=\mathbf{E}[\nabla g(\bar{x}, \xi)+\nabla g(\bar{x}, \xi)] \in$ $\partial F(\bar{x})$. Using the optimality conditions for (11) in $\bar{x}$, i.e. $0 \in \partial F(\bar{x})$, we get:

$$
F(x)-F^{*} \geq \frac{1}{2 L_{f}} \mathbf{E}\left[\|\nabla f(x, \xi)-\nabla f(\bar{x}, \xi)\|^{2}\right] .
$$

Therefore, for any $\nabla g(x, \xi) \in \partial g(x, \xi)$ we have:

$$
\begin{aligned}
\mathbf{E}\left[\|\nabla F(x, \xi)\|^{2}\right] & =\mathbf{E}\left[\|\nabla f(x, \xi)-\nabla f(\bar{x}, \xi)+\nabla g(x, \xi)+\nabla f(\bar{x}, \xi)\|^{2}\right] \\
& \leq 2 \mathbf{E}\left[\|\nabla f(x, \xi)-\nabla f(\bar{x}, \xi)\|^{2}\right]+2 \mathbf{E}\left[\|\nabla g(x, \xi)+\nabla f(\bar{x}, \xi)\|^{2}\right] \\
& \leq 4 L_{f}\left(F(x)-F^{*}\right)+2 \mathbf{E}\left[\|\nabla g(x, \xi)+\nabla f(\bar{x}, \xi)\|^{2}\right] .
\end{aligned}
$$

Assuming now that the regularization function $g(x, \xi)$ has bounded subgradients, i.e. $\|\nabla g(x, \xi)\| \leq B_{g}$, then we get that the stochastic bounded gradient condition (2) holds with:

$$
L=4 L_{f} \quad \text { and } \quad B^{2}=4\left(B_{g}^{2}+\min _{\bar{x} \in X^{*}} \mathbf{E}\left[\|\nabla f(\bar{x}, \xi)\|^{2}\right]\right) .
$$

Further, many practical problems satisfy the quadratic functional growth condition (3), the most relevant one is given next.

Example 3 [Composition between a strongly convex function and a linear map satisfy Assumption 1.2: Assume $F(x)=\hat{f}\left(A^{T} x\right)+g(x)$, where $\hat{f}$ is a strongly convex function with constant $\sigma_{f}>0, A$ is a matrix of appropriate dimension and $g$ is a polyhedral function. Since $g$ has a polyhedral epigraph, then the optimization problem (1) can be equivalently written as:

$$
\min _{x, \zeta} \hat{f}\left(A^{T} x\right)+\zeta \quad \text { s.t. : } \quad C x+c \zeta \leq d,
$$


for some appropriate matrix $C$ and vectors $c$ and $d$ of appropriate dimensions. In conclusion, this reformulation leads to the following extended problem:

$$
\hat{F}^{*}=\min _{\hat{x}=\left[x^{T} \zeta\right]^{T}} \hat{F}(\hat{x}) \quad\left(=\hat{f}(\hat{A} \hat{x})+\hat{c}^{T} \hat{x}\right) \quad \text { s.t. : } \quad \hat{C} \hat{x} \leq \hat{d},
$$

where $\hat{A}=\left[\begin{array}{ll}A & 0\end{array}\right], \hat{c}=\left[\begin{array}{ll}0 & 1\end{array}\right]^{T}, \hat{C}=\left[\begin{array}{ll}C & c\end{array}\right]$ and $\hat{d}=d$. It can be easily seen that $\hat{x}^{*}=\left[\left(x^{*}\right)^{T} \zeta^{*}\right]^{T}$ is an optimal point of this extended optimization problem if $x^{*}$ is optimal for the original problem and $g\left(x^{*}\right)=\zeta^{*}$. Moreover, we have $\hat{F}^{*}=F^{*}$. Following a standard argument, as e.g. in [8], there exist $b^{*}$ and $s^{*}$ such that the optimal set of the extended optimization problem is given by $\hat{X}^{*}=\left\{\hat{x}: \hat{A} \hat{x}=b^{*}, \hat{c}^{T} \hat{x}=s^{*}, \hat{C} \hat{x} \leq \hat{d}\right\}$. Further, since $\hat{f}$ is strongly convex function with constant $\sigma_{f}>0$, it follows from [8] [Theorem 10] that for any $M>0$ on any sublevel set defined in terms of $M$ the function $\hat{F}$ satisfies a quadratic functional growth condition of the form:

$$
\hat{F}(\hat{x})-\hat{F}^{*} \geq \frac{\mu(M)}{2}\left\|\hat{x}-\hat{x}^{*}\right\|^{2} \quad \forall \hat{x}: \hat{F}(\hat{x})-\hat{F}^{*} \leq M,
$$

where $\mu(M)=\frac{\sigma_{f}}{\theta^{2}\left(1+M \sigma_{f}+2\left\|\nabla \hat{f}\left(\hat{A} \hat{x}^{*}\right)\right\|^{2}\right)}$, with $\hat{x}^{*} \in \hat{X}^{*}$ and $\theta$ is the Hoffman bound for the optimal polyhedral set $\hat{X}^{*}$. Now, setting $\zeta=g(x)$ in the previous inequality, we get $F(x)-F(\bar{x}) \geq \frac{\mu(M)}{2}\left(\|x-\bar{x}\|^{2}+\left(\zeta-\zeta^{*}\right)^{2}\right) \geq \frac{\mu(M)}{2}\|x-\bar{x}\|^{2}$ for all $x: F(x)-F(\bar{x}) \leq M$. In conclusion, the objective function $F$ satisfies the quadratic functional growth condition (3) on any sublevel set, that is for any $M>0$ there exists $\mu(M)>0$ defined above such that:

$$
F(x)-F(\bar{x}) \geq \frac{\mu(M)}{2}\|x-\bar{x}\|^{2} \quad \forall x: F(x)-F(\bar{x}) \leq M .
$$

The quadratic functional growth condition (3) is a relaxation of strong convexity notion, see $[8$ for a more detailed discussion. Clearly, any strongly convex function $F$ satisfies (3) (see e.g. [13]).

\subsection{Preliminaries}

The following results are also useful in the sequel for proving sublinear convergence rates for the stochastic first order methods that we analyze in this paper. They are simple adaptations of some standard recurrences for $t \geq 0$ to $t \geq t_{0}$, for some finite index $t_{0}$, see e.g. 77,11,14, 16, 17.

Lemma 1.1 (Lemma 4, [16]) If there exist constants $c, d>0$ and finite index $t_{0} \geq 0$ such that the nonnegative sequence $r_{t}$ has $r_{t_{0}}$ finite and satisfies the recurrence:

$$
r_{t+1} \leq\left(1-\frac{c}{t+1}\right) r_{t}+\frac{d}{(t+1)^{2}} \quad \forall t \geq t_{0}
$$

then $r_{t}$ can be bounded as:

$$
r_{t} \leq\left\{\begin{array}{lll}
\frac{2 t_{0} r_{t_{0}}+2 d\left(1+\log \left((t+1) /\left(t_{0}+1\right)\right)\right)}{t+1} & \text { if } c=1 \\
\left(r_{t_{0}}\left(t_{0}+1\right)^{c}+\frac{2 d(2-c)\left(t_{0}+1\right)^{c}}{1-c}\right) \frac{1}{(t+1)^{c}} & \text { if } & c<1 \\
\left.\left(t_{0}+1\right) r_{t_{0}}+\frac{d}{c-1}\right) \frac{1}{t+1} & \text { if } & c>1
\end{array} \quad \forall t \geq t_{0} .\right.
$$


Lemma 1.2 (Lemma 5, [16]; Theorem 14, [14]) If there exist constants $c, d>0, \gamma \in(0,1)$ and $\zeta>\gamma$ and finite index $t_{0} \geq 0$ such that the nonnegative sequence $r_{t}$ has $r_{t_{0}}$ finite and satisfies the recurrence:

$$
r_{t+1} \leq\left(1-\frac{c}{(t+1)^{\gamma}}\right) r_{t}+\frac{d}{(t+1)^{\zeta}} \quad \forall t \geq t_{0}
$$

then $r_{t}$ can be bounded as: $r_{t} \leq \mathcal{O}\left(\frac{1}{t^{\zeta-\gamma}}\right) r_{t_{0}} \quad \forall t \geq t_{0}$.

\section{Stochastic first order methods}

Our general composite stochastic optimization problem (11) is flexible, allowing us to deal with objective functions having specific structures. Specifically, we can assume that the first term $f(\cdot, \xi)$ is given by a black-box (sub)gradient oracle or is proximal friendly. Based on these properties we propose two stochastic first order methods for solving the composite optimization problem, that is the stochastic proximal gradient and the stochastic proximal point algorithms.

\subsection{Stochastic proximal gradient (SPG)}

In this section we consider stochastic composite objective functions formed as a sum of two convex terms: first term, $f(\cdot, \xi)$, is given by a black-box (sub)gradient oracle and consequently we have access to unbiased stochastic estimates of the (sub)gradients of $f$ in the sense that $\mathbb{E}[\nabla f(x, \xi)] \in \partial f(x)$, and second term, $g(\cdot, \xi)$, admits an easily computable proximal mapping. Regularizers $g(\cdot, \xi)$ that admit closed form solution of the prox operator is e.g. Lasso type $\lambda\|x\|_{1}$ or elastic net $\lambda_{1}\|x\|^{2}+\lambda_{2}\|x\|_{1}$ for which the prox can be computed via a soft-thresholding function. Therefore, for solving the general composite problem (11) we consider the stochastic proximal gradient (SPG) algorithm:

Algorithm 2.1 Let $\left(\xi_{t}\right)_{t \in \mathbb{N}}$ be an i.i.d sequence and $x_{0} \in \mathbb{R}^{m}$. Iterate:

$$
\nabla f\left(x_{t}, \xi_{t}\right) \in \partial f\left(x_{t}, \xi_{t}\right) \quad \text { and } \quad x_{t+1}=\operatorname{prox}_{\gamma_{t} g\left(\cdot, \xi_{t}\right)}\left(x_{t}-\gamma_{t} \nabla f\left(x_{t}, \xi_{t}\right)\right),
$$

where $\left(\gamma_{t}\right)_{t \in \mathbb{N}}$ is a strictly positive sequence of stepsizes.

Note that when $g(\cdot, \xi)$ is the indicator function of a simple non-empty closed convex set $C_{\xi}$, that is $g(x, \xi)=\mathbf{1}_{C_{\xi}}(x)$, then the previous SPG algorithm becomes a stochastic projected (sub)gradient descent method [5, 11, 12]:

$$
x_{t+1}=\Pi_{C_{\xi_{t}}}\left(x_{t}-\gamma_{t} \nabla f\left(x_{t}, \xi_{t}\right)\right) .
$$

Let us define the stochastic gradient mapping (for simplicity we omit its dependence on stepsize $\gamma$ ):

$$
\mathcal{G}(x ; \xi)=\gamma^{-1}\left(x-\operatorname{prox}_{\gamma g(\cdot, \xi)}(x-\gamma \nabla f(x, \xi))\right) .
$$

Then, it follows immediately that the previous stochastic proximal gradient iteration can be written as:

$$
x_{t+1}=x_{t}-\gamma_{t} \mathcal{G}\left(x_{t} ; \xi_{t}\right) .
$$


Moreover, from optimality condition of prox operator there exists $\nabla g\left(x_{t+1}, \xi_{t}\right) \in$ $\partial g\left(x_{t+1}, \xi_{t}\right)$ such that:

$$
\mathcal{G}\left(x_{t} ; \xi_{t}\right)=\nabla f\left(x_{t}, \xi_{t}\right)+\nabla g\left(x_{t+1}, \xi_{t}\right) .
$$

Denote also $\xi_{[t]}=\left\{\xi_{0}, \ldots, \xi_{t}\right\}$. The next theorem provides a descent property for SPG and for the proof we use as main tool stochastic gradient mapping $\mathcal{G}(\cdot)$.

Theorem 2.2 Let $f(\cdot, \xi)$ and $g(\cdot, \xi)$ be convex functions such that $g$ admits an easily computable proximal operator. Additionally, assume that the stochastic bounded gradient condition from Assumption 1.1 holds. Then, for any $t \geq 0$ and stepsize $\gamma_{t}>0$, we have the following recursion for the SPG iteration:

$\mathbf{E}\left[\left\|x_{t+1}-\bar{x}_{t+1}\right\|^{2}\right] \leq \mathbf{E}\left[\left\|x_{t}-\bar{x}_{t}\right\|^{2}\right]-\gamma_{t}\left(2-\gamma_{t} L\right) \mathbf{E}\left[F\left(x_{t}\right)-F\left(\bar{x}_{t}\right)\right]+\gamma_{t}^{2} B^{2}$.

Proof From the definition of SPG iteration we have:

$$
\begin{aligned}
& \left\|x_{t+1}-\bar{x}_{t+1}\right\|^{2} \leq\left\|x_{t+1}-\bar{x}_{t}\right\|^{2}=\left\|x_{t}-\bar{x}_{t}-\gamma_{t} \mathcal{G}\left(x_{t} ; \xi_{t}\right)\right\|^{2} \\
& =\left\|x_{t}-\bar{x}_{t}\right\|^{2}-2 \gamma_{t}\left\langle\mathcal{G}\left(x_{t} ; \xi_{t}\right), x_{t}-\bar{x}_{t}\right\rangle+\gamma_{t}^{2}\left\|\mathcal{G}\left(x_{t} ; \xi_{t}\right)\right\|^{2} \\
& =\left\|x_{t}-\bar{x}_{t}\right\|^{2}-2 \gamma_{t}\left\langle\nabla f\left(x_{t}, \xi_{t}\right)+\nabla g\left(x_{t+1}, \xi_{t}\right), x_{t}-\bar{x}_{t}\right\rangle+\gamma_{t}^{2}\left\|\mathcal{G}\left(x_{t} ; \xi_{t}\right)\right\|^{2} .
\end{aligned}
$$

Now, we refine the second term. First, from convexity of $f$ we have:

$$
\left\langle\nabla f\left(x_{t}, \xi_{t}\right), x_{t}-\bar{x}_{t}\right\rangle \geq f\left(x_{t}, \xi_{t}\right)-f\left(\bar{x}_{t}, \xi_{t}\right) .
$$

Then, from convexity of $g$ and the definition of stochastic gradient mapping $\mathcal{G}(\cdot)$, we have:

$$
\begin{aligned}
& \left\langle\nabla g\left(x_{t+1}, \xi_{t}\right), x_{t}-\bar{x}_{t}\right\rangle=\left\langle\nabla g\left(x_{t+1}, \xi_{t}\right), x_{t}-x_{t+1}\right\rangle+\left\langle\nabla g\left(x_{t+1}, \xi_{t}\right), x_{t+1}-\bar{x}_{t}\right\rangle \\
& \geq \gamma_{t}\left\|\mathcal{G}\left(x_{t} ; \xi_{t}\right)\right\|^{2}-\gamma_{t}\left\langle\nabla f\left(x_{t}, \xi_{t}\right), \mathcal{G}\left(x_{t} ; \xi_{t}\right)\right\rangle+g\left(x_{t+1}, \xi_{t}\right)-g\left(\bar{x}_{t}, \xi_{t}\right) \\
& \geq \gamma_{t}\left\|\mathcal{G}\left(x_{t} ; \xi_{t}\right)\right\|^{2}-\gamma_{t}\left\langle\nabla f\left(x_{t}, \xi_{t}\right)+\nabla g\left(x_{t}, \xi_{t}\right), \mathcal{G}\left(x_{t} ; \xi_{t}\right)\right\rangle+g\left(x_{t}, \xi_{t}\right)-g\left(\bar{x}_{t}, \xi_{t}\right) .
\end{aligned}
$$

Replacing the previous two inequalities in (6), we obtain:

$$
\begin{aligned}
\left\|x_{t+1}-\bar{x}_{t+1}\right\|^{2} \leq & \left\|x_{t}-\bar{x}_{t}\right\|^{2}-2 \gamma_{t}\left(f\left(x_{t}, \xi_{t}\right)+g\left(x_{t}, \xi_{t}\right)-f\left(\bar{x}_{t}, \xi_{t}\right)-g\left(\bar{x}_{t}, \xi_{t}\right)\right) \\
& +2 \gamma_{t}^{2}\left\langle\nabla f\left(x_{t}, \xi_{t}\right)+\nabla g\left(x_{t}, \xi_{t}\right), \mathcal{G}\left(x_{t} ; \xi_{t}\right)\right\rangle-\gamma_{t}^{2}\left\|\mathcal{G}\left(x_{t} ; \xi_{t}\right)\right\|^{2} .
\end{aligned}
$$

Since $2\langle u, v\rangle-\|v\|^{2} \leq\|u\|^{2}$ for all $v \in \mathbb{R}^{n}$ and using that $F\left(x_{t}, \xi_{t}\right)=f\left(x_{t}, \xi_{t}\right)+$ $g\left(x_{t}, \xi_{t}\right)$ and $\nabla F\left(x_{t}, \xi_{t}\right)=\nabla f\left(x_{t}, \xi_{t}\right)+\nabla g\left(x_{t}, \xi_{t}\right)$, we further get:

$\left\|x_{t+1}-\bar{x}_{t+1}\right\|^{2} \leq\left\|x_{t}-\bar{x}_{t}\right\|^{2}-2 \gamma_{t}\left(F\left(x_{t}, \xi_{t}\right)-F\left(\bar{x}_{t}, \xi_{t}\right)\right)+\gamma_{t}^{2}\left\|\nabla F\left(x_{t}, \xi_{t}\right)\right\|^{2}$.

Note that $x_{t}$ depends only on history $\xi_{[t-1]}=\left\{\xi_{0}, \ldots, \xi_{t-1}\right\}$, not $\xi_{t}$. It follows from the basic property of conditional expectation that:

$$
\begin{aligned}
& \mathbf{E}_{\xi_{[t]}}\left[\left\|x_{t+1}-\bar{x}_{t+1}\right\|^{2}\right] \\
& \leq \mathbf{E}_{\xi_{[t-1]}}\left[\left\|x_{t}-\bar{x}_{t}\right\|^{2}\right]-2 \gamma_{t} \mathbf{E}_{\xi_{[t-1]}}\left[\mathbf{E}_{\xi_{t}}\left[F\left(x_{t}, \xi_{t}\right)-F\left(\bar{x}_{t}, \xi_{t}\right) \mid \xi_{[t-1]}\right]\right] \\
& \left.\quad \quad+\gamma_{t}^{2} \mathbf{E}_{\xi_{[t]}}\left[\left\|\nabla F\left(x_{t}, \xi_{t}\right)\right\|^{2}\right]\right] \\
& =\mathbf{E}_{\xi_{[t-1]}}\left[\left\|x_{t}-\bar{x}_{t}\right\|^{2}\right]-2 \gamma_{t} \mathbf{E}_{\xi_{[t-1]}}\left[F\left(x_{t}\right)-F\left(\bar{x}_{t}\right)\right]+\gamma_{t}^{2} \mathbf{E}_{\xi_{[t]}}\left[\left\|\nabla F\left(x_{t}, \xi_{t}\right)\right\|^{2}\right] .
\end{aligned}
$$


Further, making use of the stochastic bounded gradient condition given in Assumption 1.1 in the previous relation we get:

$$
\begin{aligned}
& \mathbf{E}_{\xi_{[t]}}\left[\left\|x_{t+1}-\bar{x}_{t+1}\right\|^{2}\right] \\
& \leq \mathbf{E}_{\xi_{[t-1]}}\left[\left\|x_{t}-\bar{x}_{t}\right\|^{2}\right]-2 \gamma_{t} \mathbf{E}_{\xi_{[t-1]}}\left[F\left(x_{t}\right)-F\left(\bar{x}_{t}\right)\right]+\gamma_{t}^{2} \mathbf{E}_{\xi_{[t]}}\left[\left\|\nabla F\left(x_{t}, \xi_{t}\right)\right\|^{2}\right] \\
& \stackrel{(2)}{\leq} \mathbf{E}_{\xi_{[t-1]}}\left[\left\|x_{t}-\bar{x}_{t}\right\|^{2}\right]-2 \gamma_{t} \mathbf{E}_{\xi_{[t-1]}}\left[F\left(x_{t}\right)-F\left(\bar{x}_{t}\right)\right] \\
& \quad+\gamma_{t}^{2}\left(B^{2}+L \mathbf{E}_{\xi_{[t-1]}}\left[F\left(x_{t}\right)-F\left(\bar{x}_{t}\right)\right]\right) \\
& \quad=\mathbf{E}_{\xi_{[t-1]}}\left[\left\|x_{t}-\bar{x}_{t}\right\|^{2}\right]-\gamma_{t}\left(2-\gamma_{t} L\right) \mathbf{E}_{\xi_{[t-1]}}\left[F\left(x_{t}\right)-F\left(\bar{x}_{t}\right)\right]+\gamma_{t}^{2} B^{2},
\end{aligned}
$$

which, omitting the dependence of expectation on $\xi_{[t]}$, proves the statement of the theorem.

\subsection{Stochastic proximal point (SPP)}

Flexibility in the general optimization problem (1) allow us to also consider simple proximal convex functions $f(\cdot, \xi)$ and $g(\cdot, \xi)$. In this case it is much better to use the entire function than just the gradient. Typical examples of functions $f(\cdot, \xi)$ that admit easily computable prox operators are e.g. the quadratic function $\left(z_{\xi}^{T} x-y_{\xi}\right)^{2}$ or hinge loss $\max \left(0,1-y_{\xi} z_{\xi}^{T} x\right)$ whose prox can be computed in closed form in $\mathcal{O}(n)$ operations, or logistic function $\log \left(1+e^{-y_{\xi} z_{\xi}^{T} x}\right)$ whose prox operator does not have closed form expression, but it can be computed very efficiently using Newton iteration on a univariate optimization problem. Therefore, we also present an algorithm, which we call stochastic proximal point (SPP), where at each iteration we first compute the proximal mapping with respect to the given function $f(\cdot, \xi)$ to the previous iterate and then we perform the same strategy for the regularization function $g(\cdot, \xi)$. More precisely, for solving the general composite problem (1) we consider the following SPP algorithm:

Algorithm 2.3 Let $\left(\xi_{t}\right)_{t \in \mathbb{N}}$ be an i.i.d sequence, and $x_{0} \in \mathbb{R}^{m}$. Iterate:

$$
x_{t+1 / 2}=\operatorname{prox}_{\gamma_{t} f\left(\cdot, \xi_{t}\right)}\left(x_{t}\right) \quad \text { and } \quad x_{t+1}=\operatorname{prox}_{\gamma_{t} g}\left(x_{t+1 / 2}\right) \text {, }
$$

where $\left(\gamma_{t}\right)_{t \in \mathbb{N}}$ is a strictly positive sequence of stepsizes.

Note that SPG can be viewed as SPP method applied to the linearization of $f(z ; \xi)$ in $x$, that is to the linear function: $\ell_{f}(z ; x, \xi)=f(x ; \xi)+\langle\nabla f(x ; \xi), z-$ $x\rangle$. Of course, when $f$ has an easily computable proximal operator, it is natural to use $f$ instead of its linearization $\ell_{f}$. Let us first derive some basic property for the prox operator. We first recall that any strongly convex function $h$ with convexity constant $\sigma_{h}$ and having the optimal point $x^{*}$ satisfies the following inequality [13]: $h(x) \geq h\left(x^{*}\right)+\frac{\sigma_{h}}{2}\left\|x-x^{*}\right\|^{2} \quad \forall x$. Since for any convex function $h$ and fixed point $x$, the Moreau smoothing function $h_{\gamma}(y ; x)=h(y)+\frac{1}{2 \gamma} \| y-$ 
$x \|^{2}$ is strongly convex in the first argument $y$ with strong convexity constant $1 / \gamma$, the following holds for all $x, y \in \mathbb{R}^{n}$ :

$h(y)+\frac{1}{2 \gamma}\|y-x\|^{2} \geq h\left(\operatorname{prox}_{\gamma h}(x)\right)+\frac{1}{2 \gamma}\left\|\operatorname{prox}_{\gamma h}(x)-x\right\|^{2}+\frac{1}{2 \gamma}\left\|\operatorname{prox}_{\gamma h}(x)-y\right\|^{2}$.

The next theorem provides a descent property for the SPP iteration and its proof is based on the previous Moreau smoothing condition.

Theorem 2.4 Let $f(\cdot, \xi)$ and $g(\cdot, \xi)$ be convex functions that admit easily computable proximal operators. Additionally, assume that the stochastic bounded gradient condition from Assumption 1.1 holds and $g(\cdot, \xi)$ have bounded subgradients, that is there exists $B_{g}>0$ such that $\|\nabla g(x, \xi)\| \leq B_{g}$ for all $x$ and $\xi$. Then, for any stepsize $\gamma_{t}>0$ we have the following recursive inequality for the SPP iteration:

$$
\begin{aligned}
& \mathbf{E}\left[\left\|x_{t+1}-\bar{x}_{t+1}\right\|^{2}\right] \leq \\
& \mathbf{E}\left[\left\|x_{t}-\bar{x}_{t}\right\|^{2}\right]-\gamma_{t}\left(2-\gamma_{t} L\right) \mathbf{E}\left[F\left(x_{t}\right)-F\left(\bar{x}_{t}\right)\right]+\gamma_{t}^{2}\left(B^{2}+B_{g}^{2}\right) .
\end{aligned}
$$

Proof Using (8) for the function $h=f\left(\cdot, \xi_{t}\right)$ with $y=\bar{x}_{t}$ and $x=x_{t}$ we get:

$f\left(\bar{x}_{t}, \xi_{t}\right)+\frac{1}{2 \gamma_{t}}\left\|x_{t}-\bar{x}_{t}\right\|^{2} \geq f\left(x_{t+1 / 2}, \xi_{t}\right)+\frac{1}{2 \gamma_{t}}\left\|x_{t+1 / 2}-x_{t}\right\|^{2}+\frac{1}{2 \gamma_{t}}\left\|x_{t+1 / 2}-\bar{x}_{t}\right\|^{2}$.

Using again (8) for function $h=g\left(\cdot, \xi_{t}\right)$ with $y=\bar{x}_{t}$ and $x=x_{t+1 / 2}$ we get:

$g\left(\bar{x}_{t}, \xi_{t}\right)+\frac{1}{2 \gamma_{t}}\left\|x_{t+1 / 2}-\bar{x}_{t}\right\|^{2} \geq g\left(x_{t+1}, \xi_{t}\right)+\frac{1}{2 \gamma_{t}}\left\|x_{t+1}-x_{t+1 / 2}\right\|^{2}+\frac{1}{2 \gamma_{t}}\left\|x_{t+1}-\bar{x}_{t}\right\|^{2}$.

Adding the previous two inequalities and using the convexity of $f\left(\cdot, \xi_{t}\right)$ and $g\left(\cdot, \xi_{t}\right)$, we get:

$$
\begin{aligned}
& f\left(\bar{x}_{t}, \xi_{t}\right)+g\left(\bar{x}_{t}, \xi_{t}\right)+\frac{1}{2 \gamma_{t}}\left\|x_{t}-\bar{x}_{t}\right\|^{2}-\frac{1}{2 \gamma_{t}}\left\|x_{t+1}-\bar{x}_{t}\right\|^{2} \\
& \geq f\left(x_{t+1 / 2}, \xi_{t}\right)+g\left(x_{t+1}, \xi_{t}\right)+\frac{1}{2 \gamma_{t}}\left\|x_{t+1 / 2}-x_{t}\right\|^{2}+\frac{1}{2 \gamma_{t}}\left\|x_{t+1}-x_{t+1 / 2}\right\|^{2} \\
& \geq f\left(x_{t}, \xi_{t}\right)+g\left(x_{t}, \xi_{t}\right)+\left\langle\nabla f\left(x_{t}, \xi_{t}\right)+\nabla g\left(x_{t}, \xi_{t}\right), x_{t+1 / 2}-x_{t}\right\rangle \\
& \quad \quad+\frac{1}{2 \gamma_{t}}\left\|x_{t+1 / 2}-x_{t}\right\|^{2}+\left\langle\nabla g\left(x_{t}, \xi_{t}\right), x_{t+1}-x_{t+1 / 2}\right\rangle+\frac{1}{2 \gamma_{t}}\left\|x_{t+1}-x_{t+1 / 2}\right\|^{2} \\
& \geq f\left(x_{t}, \xi_{t}\right)+g\left(x_{t}, \xi_{t}\right)-\frac{\gamma_{t}}{2}\left(\left\|\nabla f\left(x_{t}, \xi_{t}\right)+\nabla g\left(x_{t}, \xi_{t}\right)\right\|^{2}+\left\|\nabla g\left(x_{t}, \xi_{t}\right)\right\|^{2}\right),
\end{aligned}
$$

where in the last inequality we used that $\langle\alpha, z\rangle+\frac{1}{2 \gamma}\|z\|^{2} \geq-\frac{\gamma}{2}\|\alpha\|^{2}$ for all $z$. Taking now expectation and using that $\left\|x_{t+1}-\bar{x}_{t}\right\|^{2} \geq\left\|x_{t+1}-\bar{x}_{t+1}\right\|^{2}$ we get:

$$
\begin{aligned}
& \mathbf{E}_{\xi_{[t]}}\left[\left\|x_{t+1}-\bar{x}_{t+1}\right\|^{2}\right] \leq \mathbf{E}_{\xi_{[t-1]}}\left[\left\|x_{t}-\bar{x}_{t}\right\|^{2}-2 \gamma_{t} \mathbf{E}_{\xi_{[t-1]}}\left[F\left(x_{t}\right)-F\left(\bar{x}_{t}\right)\right]\right. \\
& \quad+\gamma_{t}^{2} \mathbf{E}_{\xi_{[t]}}\left[\left\|\nabla F\left(x_{t}, \xi_{t}\right)\right\|^{2}+\left\|\nabla g\left(x_{t}, \xi_{t}\right)\right\|^{2}\right]
\end{aligned}
$$

where we used basic properties of the conditional expectation as in the proof of Theorem 2.2. Further, using the stochastic bounded gradient condition (2) and omitting the dependence of expectation on $\xi_{[t]}$, we get the statement of the theorem. 
2.3 Convergence rates for stochastic first order methods

Let us denote $R_{t}^{2}=\mathbf{E}_{\xi_{[t-1]}}\left[\left\|x_{t}-\bar{x}_{t}\right\|^{2}\right]$. It follows that $R_{0}=\left\|x_{0}-\bar{x}_{0}\right\|^{2}$. From Theorem 2.2 for SPG and Theorem 2.4 for SPP, we can derive various convergence rates for the stochastic first order (SFO) methods (i.e. SPG, SPP) depending on the values taken by the constants $B, L$ and $\mu$ and also depending on the choice of the stepsize: constant or variable. Our convergence results recover, complement or extend the previous convergence rates for stochastic gradient descent (SGD) to more general functions $f(\cdot, \xi)$ and $g(\cdot, \xi)$. To derive the convergence rates for the two SFO algorithms derived in the previous sections, first we can notice that the descent relations from Theorem 2.2 and Theorem 2.4 can be written compactly as:

$\mathbf{E}\left[\left\|x_{t+1}-\bar{x}_{t+1}\right\|^{2}\right] \leq \mathbf{E}\left[\left\|x_{t}-\bar{x}_{t}\right\|^{2}\right]-\gamma_{t}\left(2-\gamma_{t} L\right) \mathbf{E}\left[F\left(x_{t}\right)-F\left(\bar{x}_{t}\right)\right]+\gamma_{t}^{2} \mathcal{B}^{2}$,

where

$$
\mathcal{B}^{2} \leq\left\{\begin{array}{lll}
B^{2} & \text { if } & \text { SPG } \\
B^{2}+B_{g}^{2} & \text { if } & \text { SPP. }
\end{array}\right.
$$

Based on this common descent property of our stochastic first order methods, for the convex case (i.e. $\mu=0$ ) and constant stepsize we get the following convergence result (we make the convention $2 / 0=\infty$ ):

Theorem 2.5 (Sublinear convergence with constant stepsize) Let assumptions of Theorem 2.2 for SPG and Theorem 2.4 for SPP hold. Additionally, we assume constant stepsizes $\gamma_{t} \equiv \gamma \in(0,2 / L)$. Then, we get the following relation for the average of iterates $\hat{x}_{t}=\frac{1}{t} \sum_{j=0}^{t-1} x_{j}$ of SFO methods (i.e. $S P G, S P P)$ in the expected value function gap:

$$
\mathbf{E}\left[F\left(\hat{x}_{t}\right)-F^{*}\right] \leq \frac{R_{0}^{2}}{t \gamma(2-\gamma L)}+\frac{\gamma \mathcal{B}^{2}}{(2-\gamma L)} \quad \forall t \geq 1 .
$$

Consequently, let $T$ the number of iterations of $S F O$ be fixed such that $T \mathcal{B}^{2}>$ $R_{0}^{2} L^{2}$ and $\gamma_{t} \equiv \gamma=R_{0} / \sqrt{T \mathcal{B}^{2}}$, then we obtain $\mathcal{O}(1 / \sqrt{T})$ convergence rate:

$$
\mathbf{E}\left[F\left(\hat{x}_{T}\right)-F^{*}\right] \leq \frac{2 R_{0} \mathcal{B}}{\sqrt{T}} .
$$

If $\mathcal{B}=0$ and $\gamma_{t} \equiv \gamma \in(0,2 / L)$, then we obtain $\mathcal{O}(1 / T)$ convergence rate:

$$
\mathbf{E}\left[F\left(\hat{x}_{T}\right)-F^{*}\right] \leq \frac{R_{0}^{2}}{T \gamma(2-\gamma L)} .
$$

Proof Adding the inequality (10) from $j=0$ to $j=t-1$ for $\gamma_{t}=\gamma$, we get:

$$
\gamma(2-\gamma L) \sum_{j=0}^{t-1} \mathbf{E}\left[F\left(x_{j}\right)-F\left(\bar{x}_{j}\right)\right] \leq \mathbf{E}\left[\left\|x_{0}-\bar{x}_{0}\right\|^{2}\right]-\mathbf{E}\left[\left\|x_{t}-\bar{x}_{t}\right\|^{2}\right]+t \gamma^{2} \mathcal{B}^{2} .
$$


Using Jensen's inequality for the convex function $F$ and $\gamma \in(0,2 / L)$, we get:

$$
\begin{aligned}
\gamma(2-\gamma L) t \mathbf{E}\left[F\left(\hat{x}_{t}\right)-F^{*}\right] & \leq \gamma(2-\gamma L) t \mathbf{E}\left[\sum_{j=0}^{t-1} \frac{1}{t} F\left(x_{j}\right)-F^{*}\right] \\
& \leq\left\|x_{0}-\bar{x}_{0}\right\|^{2}+\gamma^{2} t \mathcal{B}^{2},
\end{aligned}
$$

which leads immediately to our first statement. Fixing the number of iteration $T$ and minimizing the right hand side in (11) w.r.t. $\gamma$ we obtain the best stepsize of the form $\mathcal{O}(1 / \sqrt{T})$ and the corresponding convergence rate $\mathcal{O}(1 / \sqrt{T})$. However, the resulting expressions are cumbersome. On the other hand, if $T \mathcal{B}^{2}>R_{0}^{2} L^{2}$ and consider the stepsize $\gamma=R_{0} / \sqrt{T \mathcal{B}^{2}}$ we get a simpler expression for the convergence rate. Indeed, for these choices we have that $2-\gamma L \geq 1$ and using this in the inequality (12), we obtain $\mathbf{E}\left[F\left(\hat{x}_{T}\right)-F^{*}\right] \leq \frac{2 R_{0} \mathcal{B}}{\sqrt{T}}$. Convergence rate $\mathcal{O}(1 / T)$ for $\mathcal{B}=0$ follows immediately from (12).

Theorem 2.6 (Sublinear convergence with variable stepsize) Let assumptions of Theorem 2.2 for SPG and Theorem 2.4 for SPP hold. Additionally, we assume $L>0$, variable stepsize $\gamma_{t}=\gamma_{0} / \sqrt{t}$ with $\gamma_{0}=1 / L$ and the distance of the iterates to the optimal set is bounded almost surely, i.e. $\mathbf{E}\left[\left\|x_{t}-\bar{x}_{t}\right\|^{2}\right] \leq R^{2}$ for all $t \geq 0$. Then, we get the following rate for the average of iterates $\hat{x}_{t}=\frac{1}{t} \sum_{j=0}^{t-1} x_{j}$ of SFO in the expected value function gap:

$$
\mathbf{E}\left[F\left(\hat{x}_{t}\right)-F^{*}\right] \leq \frac{1}{\sqrt{t}}\left(R^{2} L+\frac{2 \mathcal{B}^{2}}{L}\right) \quad \forall t \geq 1 .
$$

Proof Since $\gamma_{t}=\gamma_{0} / \sqrt{t}$ with $\gamma_{0}=1 / L$ it follows that $\gamma_{t}\left(2-\gamma_{t} L\right) \geq \gamma_{t}$, which combined with (10) yields:

$$
\mathbf{E}\left[F\left(x_{t}\right)-F^{*}\right] \leq \frac{1}{\gamma_{t}} \mathbf{E}\left[x_{t}-\bar{x}_{t} \|^{2}\right]-\frac{1}{\gamma_{t}} \mathbf{E}\left[\|\| x_{t+1}-\bar{x}_{t+1} \|^{2}\right]+\gamma_{t} \mathcal{B}^{2}
$$

Summing up from $j=0$ to $t-1$ and using that $\gamma_{t}$ is a nonincreasing sequence, we have:

$$
\begin{aligned}
& \sum_{j=0}^{t-1} \mathbf{E}\left[F\left(x_{j}\right)-F^{*}\right] \leq \frac{1}{\gamma_{0}}\left\|x_{0}-\bar{x}_{0}\right\|^{2}+\sum_{j=0}^{t-2}\left(\frac{1}{\gamma_{j+1}}-\frac{1}{\gamma_{j}}\right) \mathbf{E}\left[\left\|x_{j+1}-\bar{x}_{j+1}\right\|^{2}\right] \\
& \quad-\frac{1}{\gamma_{t-1}} \mathbf{E}\left[\left\|x_{t}-\bar{x}_{t}\right\|^{2}\right]+\mathcal{B}^{2} \sum_{j=0}^{t-1} \gamma_{j} \\
& \leq \frac{1}{\gamma_{0}}\left\|x_{0}-\bar{x}_{0}\right\|^{2}+\sum_{j=0}^{t-2}\left(\frac{1}{\gamma_{j+1}}-\frac{1}{\gamma_{j}}\right) \mathbf{E}\left[\left\|x_{j+1}-\bar{x}_{j+1}\right\|^{2}\right]+\mathcal{B}^{2} \sum_{j=0}^{t-1} \gamma_{j} \\
& \leq \frac{R^{2}}{\gamma_{0}}+\sum_{j=0}^{t-2}\left(\frac{1}{\gamma_{j+1}}-\frac{1}{\gamma_{j}}\right) R^{2}+\mathcal{B}^{2} \sum_{j=0}^{t-1} \gamma_{j}=\frac{R^{2}}{\gamma_{0}}+\left(\frac{1}{\gamma_{t-1}}-\frac{1}{\gamma_{0}}\right) R^{2}+\mathcal{B}^{2} \sum_{j=0}^{t-1} \gamma_{j} \\
& =\frac{R^{2}}{\gamma_{t-1}}+\mathcal{B}^{2} \sum_{j=0}^{t-1} \gamma_{j} .
\end{aligned}
$$


Using now the Jensen's inequality for the convex function $F$, we get:

$$
\mathbf{E}\left[F\left(\hat{x}_{t}\right)-F^{*}\right] \leq \frac{R^{2}}{t \gamma_{t-1}}+\frac{\mathcal{B}^{2}}{t} \sum_{j=0}^{t-1} \gamma_{j} \leq \frac{R^{2} L}{\sqrt{t}}+\frac{2 \mathcal{B}^{2}}{L \sqrt{t}},
$$

which proves our statement.

If we assume additionally some quadratic functional growth condition (i.e. $\mu>0$ ) and run the two SFO methods with constant stepsize, the next theorem proves that we can achieve linear convergence to a noise dominated region proportional to the stepsize and in some cases even pure linear convergence provided that $\mathcal{B}=0$.

Theorem 2.7 (Sufficient conditions for linear convergence of SFO) Let assumptions of Theorem 2.2 for SPG and Theorem 2.4 for SPP hold. Additionally, we assume that the quadratic functional growth condition from Assumption 1.2 holds and we choose constant stepsize $\gamma_{t}=\gamma \in(0,2 / L)$. Then, we get the following upper bounds for the square distance of the SFO iterates to the solution set:

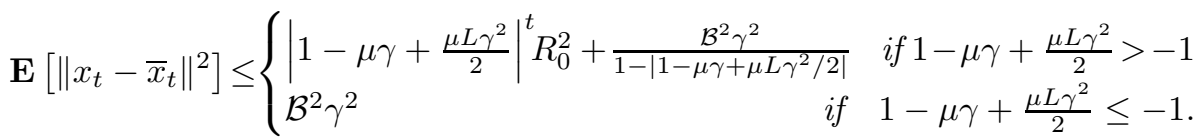

Moreover, if the condition $\mu<4 L$ holds or the constant stepsize satisfies $\gamma<\min (2 / L, 2 / \mu)$, then $\left|1-\mu \gamma+\frac{\mu L \gamma^{2}}{2}\right|<1$ and therefore we get linear converge to a noise dominated region with radius proportional to the square of the stepsize $\gamma$ :

$$
\mathbf{E}\left[\left\|x_{t}-\bar{x}_{t}\right\|^{2}\right] \leq\left|1-\mu \gamma+\frac{\mu L \gamma^{2}}{2}\right|^{t} R_{0}^{2}+\frac{\mathcal{B}^{2} \gamma^{2}}{1-\left|1-\mu \gamma+\mu L \gamma^{2} / 2\right|} .
$$

Consequently, if $\mathcal{B}=0$, then we get pure linear convergence:

$$
\mathbf{E}\left[\left\|x_{t}-\bar{x}_{t}\right\|^{2}\right] \leq\left|1-\mu \gamma+\frac{\mu L \gamma^{2}}{2}\right|^{t} R_{0}^{2}
$$

Proof From (10) and Assumption 1.2 it follows that:

$$
\begin{aligned}
& \mathbf{E}_{\xi_{[t]}}\left[\left\|x_{t+1}-\bar{x}_{t+1}\right\|^{2}\right] \leq \mathbf{E}_{\xi_{[t-1]}}\left[\left\|x_{t}-\bar{x}_{t}\right\|^{2}\right]-\gamma_{t}\left(2-\gamma_{t} L\right) \mathbf{E}_{\xi_{[t-1]}}\left[F\left(x_{t}\right)-F\left(\bar{x}_{t}\right)\right] \\
& +\mathcal{B}^{2} \gamma_{t}^{2} \stackrel{\sqrt[3]{3}}{\leq} \mathbf{E}_{\xi_{[t-1]}}\left[\left\|x_{t}-\bar{x}_{t}\right\|^{2}\right]-\frac{\mu \gamma_{t}\left(2-\gamma_{t} L\right)}{2} \mathbf{E}_{\xi_{[t-1]}}\left[\left\|x_{t}-\bar{x}_{t}\right\|^{2}\right]+\mathcal{B}^{2} \gamma_{t}^{2}
\end{aligned}
$$

provided that $\gamma_{t} \leq 2 / L$. In conclusion, we obtain the following recursion:

$$
\mathbf{E}_{\xi_{[t]}}\left[\left\|x_{t+1}-\bar{x}_{t+1}\right\|^{2}\right] \leq\left(1-\mu \gamma_{t}+\frac{\mu L \gamma_{t}^{2}}{2}\right) \mathbf{E}_{\xi_{[t-1]}}\left[\left\|x_{t}-\bar{x}_{t}\right\|^{2}\right]+\mathcal{B}^{2} \gamma_{t}^{2} .
$$


For constant stepsize $\gamma_{t}=\gamma<2 / L$ it follows that $1-\mu \gamma+\mu L \gamma^{2} / 2<1$. Then, combining inequality (14) with condition $1-\mu \gamma+\mu L \gamma^{2} / 2>-1$, we get:

$$
\begin{aligned}
\mathbf{E}\left[\left\|x_{t}-\bar{x}_{t}\right\|^{2}\right] & \leq\left|1-\mu \gamma+\frac{\mu L \gamma^{2}}{2}\right|^{t}\left\|x_{0}-\bar{x}_{0}\right\|^{2}+\mathcal{B}^{2} \gamma^{2} \sum_{j=0}^{t-1}\left|1-\mu \gamma+\frac{\mu L \gamma^{2}}{2}\right|^{j} \\
& \leq\left|1-\mu \gamma+\frac{\mu L \gamma^{2}}{2}\right|^{t}\left\|x_{0}-\bar{x}_{0}\right\|^{2}+\frac{\mathcal{B}^{2} \gamma^{2}}{1-\left|1-\mu \gamma+\mu L \gamma^{2} / 2\right|}
\end{aligned}
$$

where in the second inequality we used that $\sum_{j=0}^{t-1} c^{j} \leq \sum_{j=0}^{\infty} c^{j}=\frac{1}{1-c}$ for any $|c|<1$. Otherwise, if $1-\mu \gamma+\mu L \gamma^{2} / 2 \leq-1$, then from (14) it follows that:

$$
\mathbf{E}\left[\left\|x_{t}-\bar{x}_{t}\right\|^{2}\right] \leq \mathcal{B}^{2} \gamma^{2}
$$

Moreover, if $\mu<4 L$, then the relation $1-\mu \gamma+\mu L \gamma^{2} / 2>-1$ always holds, regardless of the value of $\gamma$. Thus, if $\mu<4 L$ and the constant stepsize is chosen such $\gamma<\frac{2}{L}$, then $\left|1-\mu \gamma+\mu L \gamma^{2}\right|<1$. Similarly, if the constant stepsize satisfies $\gamma<\min (2 / L, 2 / \mu)$, then again we have $0<1-\mu \gamma+\mu L \gamma^{2}<1$. Therefore, these two previous conditions guarantee linear convergence to a noise dominated region whose radius is proportional to the square of the stepsize $\gamma$. Finally, the inequality (14) with $\mathcal{B}=0$ and $\left|1-\mu \gamma+\mu L \gamma^{2}\right|<1$ proves linear convergence for $\mathbf{E}\left[\left\|x_{t}-\bar{x}_{t}\right\|^{2}\right]$, with $x_{t}$ generated by SFO methods.

Remark 2.1 Note that if $f$ is strongly convex and with Lipschitz continuous gradient, then the condition $\mu<4 L$ holds. Indeed, assume that the $\mu$ strongly convex function $f(\cdot, \xi)$ has $L_{f}$ Lipschitz continuous gradient and the regularization function $g$ has bounded subgradients. Then, we have:

$\left\|\nabla f(x, \xi)-\nabla f\left(x^{*}, \xi\right)\right\| \leq L_{f}\left\|x-x^{*}\right\| \quad$ and $\quad\|\nabla g(x, \xi)(x)\| \leq B_{g} \quad \forall x \in \operatorname{dom} F$,

where $x^{*}$ is the unique solution of (1). In this case it follows that:

$\|\nabla f(x, \xi)+\nabla g(x, \xi)\| \leq\left\|\nabla f(x, \xi)-\nabla f\left(x^{*}, \xi\right)\right\|+\left\|\nabla f\left(x^{*}, \xi\right)+\nabla g(x, \xi)\right\|$
$\leq L_{f}\left\|x-x^{*}\right\|+\left\|\nabla f\left(x^{*}, \xi\right)\right\|+\|\nabla g(x, \xi)\| \leq L_{f}\left\|x-x^{*}\right\|+\left(\left\|\nabla f\left(x^{*}, \xi\right)\right\|+B_{g}\right)$.

Now, since $f$ is strongly convex, it follows that $F(x)-F^{*} \geq \frac{\mu}{2}\left\|x-x^{*}\right\|^{2}$, which inserted in the previous inequality, yields:

$$
\|\nabla f(x, \xi)+\nabla g(x, \xi)\| \leq L_{f}\left(\frac{2\left(F(x)-F^{*}\right)}{\mu}\right)^{1 / 2}+\left(\left\|\nabla f\left(x^{*}, \xi\right)\right\|+B_{g}\right) .
$$

Squaring the fist and the last term in the previous relation and taking expectation we get that the stochastic bounded gradient condition (2) holds with: $L=\frac{4 L_{f}^{2}}{\mu}>\frac{\mu}{4}$, since we always have the relation $L_{f} \geq \mu$ between the Lipschitz and strong convexity constants of a convex function. 
Theorem 2.7 states that SFO methods achieve linear convergence when $B=0$ in (2) and in this case we say that the objective function $F$ satisfies a strong stochastic bounded gradient condition. Next theorem derives also necessary conditions to achieve linear convergence for SFO methods with constant stepsize. Paper [8] proves that the class of objective functions having Lipschitz continous gradient and satisfying a quadratic functional growth is the largest one for which deterministic gradient method converges linearly. Using a similar reasoning as in [8], we expect additional necessary conditions for linear convergence of SFO methods as described in the next result.

Theorem 2.8 (Necessary conditions for linear convergence of SFO) Assume that $g \equiv 0$ and $f$ satisfies a quadratic functional growth condition (Assumption 1.2) and has Lipschitz continuous gradient. Assume further that there exists $q<1$ such that the SFO iterates with constant stepsize $\gamma>0$ converge linearly, i.e. $\mathbf{E}_{\xi_{t}}\left[\left\|x_{t+1}-\bar{x}_{t+1}\right\|^{2} \mid \xi_{[t-1]}\right] \leq q\left\|x_{t}-\bar{x}_{t}\right\|^{2}$ for all $t \geq 0$, and the stochastic process $\left(x_{t}\right)_{t \geq 0}$ of SFO yields the same projection onto the optimal set, i.e. $\bar{x}_{t}=\bar{x}_{0}$ for all $t \geq 0$ almost surely. Then, the stochastic bounded gradient condition from Assumption (1.1) holds with $B \equiv 0$, or equivalently, all the partial functions $f(\cdot, \xi)$ must have the same minimizer.

Proof Note that for $g=0$ the SPG iterates coincide with the SGD updates: $x_{t+1}=x_{t}-\gamma \nabla f\left(x_{t}, \xi_{t}\right)$. Then, using that the stochastic process $\left(x_{t}\right)_{t \geq 0}$ yields the same projection onto the optimal set, i.e. $\bar{x}_{t}=\bar{x}_{0}$ for all $t \geq 0$, we get:

$$
\begin{aligned}
& \gamma^{2}\left\|\nabla f\left(x_{t}, \xi_{t}\right)\right\|^{2}=\left\|x_{t+1}-x_{t}\right\|^{2} \\
& \leq 2\left\|x_{t+1}-\bar{x}_{t}\right\|^{2}+2\left\|x_{t}-\bar{x}_{t}\right\|^{2}=2\left\|x_{t+1}-\bar{x}_{0}\right\|^{2}+2\left\|x_{t}-\bar{x}_{0}\right\|^{2} .
\end{aligned}
$$

Taking expectation with respect to $\xi_{t}$ and using $\mathbf{E}_{\xi_{t}}\left[\left\|x_{t+1}-\bar{x}_{t+1}\right\|^{2} \mid \xi_{[t-1]}\right] \leq$ $q\left\|x_{t}-\bar{x}_{t}\right\|^{2}$ and the quadratic functional growth condition (3) on $f$, we obtain:

$$
\mathbf{E}_{\xi_{t}}\left[\left\|\nabla f\left(x_{t}, \xi_{t}\right)\right\|^{2} \mid \xi_{[t-1]}\right] \leq \frac{2(1+q)}{\gamma^{2}}\left\|x_{t}-\bar{x}_{0}\right\|^{2} \leq \frac{4(1+q)}{\mu \gamma^{2}}\left(f\left(x_{t}\right)-f\left(\bar{x}_{0}\right)\right) .
$$

Similarly, for SPP we have from the definition of the proximity operator:

$$
x_{t}-x_{t+1}=\gamma \nabla f\left(x_{t+1}, \xi_{t}\right)=\gamma \nabla f\left(x_{t}, \xi_{t}\right)+\gamma \nabla f\left(x_{t+1}, \xi_{t}\right)-\gamma \nabla f\left(x_{t}, \xi_{t}\right) .
$$

Therefore, from the $L_{f}$ Lipschitz continuity of $\nabla f(\cdot, \xi)$, we get

$$
\begin{aligned}
& \gamma^{2}\left\|\nabla f\left(x_{t}, \xi_{t}\right)\right\|^{2}=\left\|x_{t}-x_{t+1}\right\|^{2}+2 \gamma\left\langle x_{t}-x_{t+1}, \nabla f\left(x_{t}, \xi_{t}\right)-\nabla f\left(x_{t+1}, \xi_{t}\right)\right\rangle \\
& \quad+\gamma^{2}\left\|\nabla f\left(x_{t+1}, \xi_{t}\right)-\nabla f\left(x_{t}, \xi_{t}\right)\right\|^{2} \leq\left(1+2 \gamma L_{f}+\gamma^{2} L_{f}\right)\left\|x_{t}-x_{t+1}\right\|^{2} \\
& \leq 2\left(1+2 \gamma L_{f}+\gamma^{2} L_{f}\right)\left(\left\|x_{t+1}-\bar{x}_{t}\right\|^{2}+\left\|x_{t}-\bar{x}_{t}\right\|^{2} .\right.
\end{aligned}
$$

Now, taking expectation on the both sides of above inequality, using that the stochastic process $\left(x_{t}\right)_{t \geq 0}$ produces the same projection onto the optimal set, i.e. $\bar{x}_{t}=\bar{x}_{0}$ for all $t \geq 0$, and $\mathbf{E}_{\xi_{t}}\left[\left\|x_{t+1}-\bar{x}_{t+1}\right\|^{2} \mid \xi_{[t-1]}\right] \leq q\left\|x_{t}-\bar{x}_{t}\right\|$, we get:

$$
\begin{aligned}
& \gamma^{2} \mathbf{E}_{\xi_{t}}\left[\left\|\nabla f\left(x_{t}, \xi_{t}\right)\right\|^{2} \mid \xi_{[t-1]}\right] \leq 2\left(1+2 \gamma L_{f}+\gamma^{2} L_{f}\right)(1+q)\left\|x_{t}-\bar{x}_{0}\right\|^{2} \\
& \leq(4 / \mu)\left(1+2 \gamma L_{f}+\gamma^{2} L_{f}\right)(1+q)\left(f\left(x_{t}\right)-f\left(\bar{x}_{0}\right)\right)
\end{aligned}
$$


where in the last inequality we used the quadratic functional growth condition (3) on $f$. On the other hand, if $f(\cdot, \xi)$ has $L_{f}$ Lipschitz continuous gradient, then for any $x \in \operatorname{dom} F$ we have:

$$
\begin{aligned}
& \|\nabla f(x, \xi)\|^{2} \leq 2\left\|\nabla f(x, \xi)-\nabla f\left(\bar{x}_{0}, \xi\right)\right\|^{2}+2\left\|\nabla f\left(\bar{x}_{0}, \xi\right)\right\|^{2} \\
& \leq 2 L_{f}^{2}\left\|x-\bar{x}_{0}\right\|^{2}+2\left\|\nabla f\left(\bar{x}_{0}, \xi\right)\right\|^{2} \leq \frac{4 L_{f}^{2}}{\mu}\left(f(x)-f\left(\bar{x}_{0}\right)\right)+2\left\|\nabla f\left(\bar{x}_{0}, \xi\right)\right\|^{2},
\end{aligned}
$$

where in the last inequality we used again the quadratic functional growth condition (3) on $f$. Taking now expectation with respect to $\xi$, we obtain:

$$
\mathbf{E}_{\xi}\left[\|\nabla f(x, \xi)\|^{2} \leq \frac{4 L_{f}^{2}}{\mu}\left(f(x)-f\left(\bar{x}_{0}\right)\right)+2 \mathbf{E}_{\xi}\left[\left\|\nabla f\left(\bar{x}_{0}, \xi\right)\right\|^{2}\right] \forall x \in \operatorname{dom} F .\right.
$$

Combining (15) or (16) with (17), it follows that $B=2 \mathbf{E}_{\xi}\left[\left\|\nabla f\left(\bar{x}_{0}, \xi\right)\right\|^{2}\right]=0$, i.e. the strong stochastic bounded gradient condition holds in (2). This shows that if SFO iterates converge linearly on the class of objective functions having Lipschitz gradient and quadratic functional growth, then the partial functions $f(\cdot, \xi)$ must have the same minimizer, i.e. $\nabla f\left(\bar{x}_{0}, \xi\right)=0$ alsmost surely.

In the next theorem we show that SFO algorithms based on a hybrid strategy consisting of using constant stepsize $\gamma=1 / L$ at the beginning and then at some well-defined iteration switching to a variable stepsize $\gamma=\mathcal{O}(1 / t)$ has a sublinear convergent behavior of order $\mathcal{O}(1 / t)$.

Theorem 2.9 (Sublinear convergence with constant-variable stepsize) Let Assumptions 1.1 and Assumption 1.2 hold, and choose variable stepsize $\gamma_{t}=\min \left(\frac{1}{L}, \frac{c}{(t+1)}\right)$, for some fixed $c>0$. Set $d=c^{2} \mathcal{B}^{2}$ and $t_{0}=\lfloor c L\rfloor$. Then, we get the following sublinear convergence rate for the square distance of the SFO iterates to the solution set:

$$
\begin{aligned}
& \mathbf{E}\left[\left\|x_{t}-\bar{x}_{t}\right\|^{2}\right] \\
& \leq \begin{cases}\frac{2 t_{0} R_{t_{0}}^{2}+2 d\left(1+\log \left((t+1) /\left(t_{0}+1\right)\right)\right)}{t+1} & \text { if } c \mu=2 \& t>t_{0}, \\
\left(R_{t_{0}}^{2}\left(1+t_{0}\right)^{0.5 c \mu}+\frac{2 d(2-0.5 c \mu)\left(t_{0}+1\right)^{0.5 c \mu}}{1-0.5 c \mu}\right) \frac{1}{(t+1)^{0.5 c \mu}} & \text { if } \quad c \mu<2 \& t>t_{0}, \\
\left.\left(t_{0}+1\right) R_{t_{0}}^{2}+\frac{d}{0.5 c \mu-1}\right) \frac{1}{t+1} & \text { if } \quad c \mu>2 \& t>t_{0} \\
\max \left(\left|1-\frac{\mu}{2 L}\right|^{t} R_{0}^{2}+\frac{\mathcal{B}^{2}}{L^{2}-\left|L^{2}-\mu L / 2\right|}, \mathcal{B}^{2} / L^{2}\right) & \text { if } t \leq t_{0} .\end{cases}
\end{aligned}
$$

Proof Since for $t \leq t_{0}$ the stepsize is $\gamma_{t}=1 / L$, it follows from Theorem 2.7 that $\mathbf{E}_{\xi_{\left[t_{0}-1\right]}}\left[\left\|x_{t_{0}}-\bar{x}_{t_{0}}\right\|^{2}\right]$ is bounded for example by:

$$
\mathbf{E}_{\xi_{\left[t_{0}-1\right]}}\left[\left\|x_{t_{0}}-\bar{x}_{t_{0}}\right\|^{2}\right] \leq \max \left(\left|1-\frac{\mu}{2 L}\right|{ }^{t_{0}} R_{0}^{2}+\frac{\mathcal{B}^{2}}{L^{2}-\left|L^{2}-\mu L / 2\right|}, \mathcal{B}^{2} / L^{2}\right) .
$$

Moreover, for our hybrid choice of the stepsizes $\gamma_{t}=\min \left(\frac{1}{L}, \frac{c}{(t+1)}\right)$ we always have $2-\gamma_{t} L \geq 1$ and consequently combining (5) with Assumption 1.2 it 
follows that:

$$
\begin{aligned}
& \mathbf{E}_{\xi_{[t]}}\left[\left\|x_{t+1}-\bar{x}_{t+1}\right\|^{2}\right] \\
& \leq \mathbf{E}_{\xi_{[t-1]}}\left[\left\|x_{t}-\bar{x}_{t}\right\|^{2}\right]-\gamma_{t}\left(2-\gamma_{t} L\right) \mathbf{E}_{\xi_{[t-1]}}\left[F\left(x_{t}\right)-F\left(\bar{x}_{t}\right)\right]+\mathcal{B}^{2} \gamma_{t}^{2} \\
& \stackrel{(3)}{\leq} \mathbf{E}_{\xi_{[t-1]}}\left[\left\|x_{t}-\bar{x}_{t}\right\|^{2}\right]-\frac{\mu \gamma_{t}\left(2-\gamma_{t} L\right)}{2} \mathbf{E}_{\xi_{[t-1]}}\left[\left\|x_{t}-\bar{x}_{t}\right\|^{2}\right]+\mathcal{B}^{2} \gamma_{t}^{2} \\
& \leq \mathbf{E}_{\xi_{[t-1]}}\left[\left\|x_{t}-\bar{x}_{t}\right\|^{2}\right]-\frac{\mu \gamma_{t}}{2} \mathbf{E}_{\xi_{[t-1]}}\left[\left\|x_{t}-\bar{x}_{t}\right\|^{2}\right]+\mathcal{B}^{2} \gamma_{t}^{2} \\
& =\left(1-\frac{c \mu}{2(t+1)}\right) \mathbf{E}_{\xi_{[t-1]}}\left[\left\|x_{t}-\bar{x}_{t}\right\|^{2}\right]+\frac{c^{2} \mathcal{B}^{2}}{(t+1)^{2}} \quad \forall t \geq t_{0} .
\end{aligned}
$$

Therefore, we obtain the recurrence $r_{t+1} \leq\left(1-\frac{c \mu}{2(t+1)}\right) r_{t}+\frac{c^{2} \mathcal{B}^{2}}{(t+1)^{2}}$ and then using Lemma 1.1 we get our statements.

From previous theorem we observe that the best convergence rate $\mathcal{O}(1 / t)$ is obtained when the constant $c$ is proportional to the inverse of the strong convexity constant $\mu$, and thus the switching time $t_{0}$ has to be about two times the condition number $L / \mu$ of the optimization problem (1), i.e.:

$$
c \approx \frac{2}{\mu} \quad \text { and } \quad t_{0} \approx 2 \frac{L}{\mu} .
$$

Remark 2.2 The previous convergence results for SFO algorithms can be easily extended to a more general and robust variable stepsize of the form:

$$
\gamma_{t}=\min \left(\frac{1}{L}, \frac{c}{(t+1)^{\alpha}}\right)
$$

for some fixed $c>0$ and $\alpha \in(0,1)$. In this case we can use Lemma 1.2 (see also [7,14]) for the recurrence:

$$
r_{t+1} \leq\left(1-\frac{c \mu}{2(t+1)^{\alpha}}\right) r_{t}+\frac{c^{2} \mathcal{B}^{2}}{(t+1)^{2 \alpha}} \quad \forall t \geq t_{0}
$$

to obtain sublinear convergence rate of order $\mathcal{O}\left(1 / t^{\alpha}\right)$ for the square distance of the SFO iterates to the solution set.

\subsection{Stochastic gradient descent revisited}

Note that our framework based on stochastic gradient mapping and Moreau mapping allows us to generalize the existing results [11]19] for stochastic gradient descent (SGD), i.e. when $g$ is the indicator function of a simple convex set $C$, to the general case of stochastic first order (SFO) algorithms that are able to deal with any convex family of functions $g(\cdot, \xi)$ that admit a tractable proximal operator. In particular, when considering the stochastic convex problem $\min _{x \in C} \mathbf{E}[f(x, \xi)]$, we recover the basic results for the SGD algorithm:

$$
(S G D): \quad x_{t+1}=\Pi_{C}\left(x_{t}-\gamma_{t} \nabla f\left(x_{t}, \xi_{t}\right)\right) .
$$


More precisely, it follows that the conclusions of Theorems 2.2, 2.5 and 2.6 are still valid provided that Assumption 1.1 is replaced with:

$$
B^{2}+L(f(x)-f(\bar{x})) \geq \mathbf{E}\left[\|\nabla f(x, \xi)\|^{2}\right] \quad \forall x \in C .
$$

Moreover, Theorems 2.7 and 2.8 hold, when Assumption 1.2 is replaced with:

$$
f(x)-f(\bar{x}) \geq \frac{\mu}{2}\|x-\bar{x}\|^{2} \quad \forall x \in C .
$$

In particular, from Theorem 2.7 under the conditions (18) with $B=0$ and (19) we get linear convergence for SGD. Note that our strong stochastic bounded gradient condition, i.e. (18) with $B=0$, may be more general than the one imposed in [19] to obtain linear convergence for SGD . More precisely, [19] considers $C=\mathbb{R}^{n}$ and the strongly convex function $f$ with Lipschitz gradient. Besides these assumptions [19] also imposes a strong growth condition:

$$
L_{\mathrm{sg}} \mathbf{E}\left[\|\nabla f(x, \xi)\|^{2}\right] \leq\|\mathbf{E}[\nabla f(x, \xi)]\|^{2} \quad \forall x \in \mathbb{R}^{n},
$$

for some $L_{\mathrm{sg}}>0$. Clearly, if the strong growth condition holds and $f$ has gradient Lipschitz with constant $L_{f}$, then (18) also holds with $B=0$, since for the unconstrained case, requirement that $f$ has gradient Lipschitz leads to:

$$
\begin{aligned}
& f(x) \geq f(\bar{x})+\langle\nabla f(\bar{x}), x-\bar{x}\rangle+\frac{1}{2 L}\|\nabla f(x)-\nabla f(\bar{x})\|^{2} \\
& \stackrel{\nabla f(\bar{x})=0}{=} f(\bar{x})+\frac{1}{2 L}\|\mathbf{E}[\nabla f(x, \xi)]\|^{2} \geq f(\bar{x})+\frac{L_{\mathrm{sg}}}{2 L} \mathbf{E}\left[\|\nabla f(x, \xi)\|^{2}\right] .
\end{aligned}
$$

Note that the conditions (18) with $B=0$ and (19) are satisfied in some applications. For example, consider the problem of finding a point in the (in)finite intersection of a family of simple closed convex sets, i.e. find $x \in C=\cap_{\xi \in \Omega} C_{\xi}$. Assume that linear regularity holds for the sets $\left(C_{\xi}\right)_{\xi \in \Omega}$, i.e. there exists finite constant $\kappa>0$ such that:

$$
\kappa\left\|x-\Pi_{C}(x)\right\|^{2} \leq \mathbf{E}\left[\left\|x-\Pi_{C_{\xi}}(x)\right\|^{2}\right] \quad \forall x \in \mathbb{R}^{n} .
$$

Typical examples of sets satisfying linear regularity are polyhedra sets or convex sets with nonempty interior $[9$. For example, when solving linear systems $A x=b$ we can take $C_{\xi}=\left\{x: a_{\xi}^{T} x=b_{\xi}\right\}$, where $a_{\xi}$ is the $\xi$ th row of matrix $A \in \mathbb{R}^{m \times n}$. In this case, $\kappa=m / \lambda_{\text {min }}^{\mathrm{nz}}\left(A^{T} A\right)$, provided that we consider a uniform probability distribution on $\Omega=\{1, \cdots, m\}$, where $\lambda_{\min }^{\mathrm{nz}}\left(A^{T} A\right)$ denotes the smallest nonzero eigenvalue of $A^{T} A$. Then, under linear regularity the convex feasibility problem can be equivalently written as a stochastic optimization problem, see also [9]:

$$
\min _{x \in \mathbb{R}^{m}} \mathbf{E}[f(x, \xi)] \quad\left(\stackrel{\text { def }}{=} \frac{1}{2} \mathbf{E}\left[\left\|x-\Pi_{C_{\xi}}(x)\right\|^{2}\right]\right) .
$$

Then, if $C \neq \emptyset$, it follows immediately that $f(x)=\mathbf{E}[f(x, \xi)]$ satisfies condition (18) with $L=2$ and $B=0$, since the following relations hold: $2 f(x)=$ $\mathbf{E}\left[\|\nabla f(x, \xi)\|^{2}\right]$ and $f(\bar{x})=0$. Moreover, it is easy to see that the linear regularity (20) can be written as (19) with $\mu=\kappa$. In conclusion, from the results 
of the previous section we have that SGD or equivalently the stochastic alternating projection algorithm $x_{t+1}=x_{t}-\gamma_{t}\left(x_{t}-\Pi_{C_{\xi_{t}}}\left(x_{t}\right)\right)$ converges linearly. Note that for linear systems $A x=b$ the previous alternating projection scheme with $\gamma_{t}=1$ becomes Kaczmarz algorithm. Similar linear convergence rates for the alternating projection has been derived in 9] and those results were the main motivation for this paper, that is extending the linear rates for stochastic alternating projection to more general stochastic problems and algorithms.

\section{Restarted stochastic first order methods}

From previous sections we observe that linear convergence can be achieved by stochastic first order (SFO) methods (i.e. SPG and SPP) provided that $B=0$ in Assumption 1.1 and Assumption 1.2. However, this condition restricts the class of functions for which we can achieve linear convergence for SFO. In this section we propose a restarting variant of SFO and prove that this new method can achieve linear convergence for classes of functions for which $B \neq 0$. This restarting variant consists of running the SPG/SPP algorithms (as a routine) for multiple times (epochs) and restarting it each time after a certain number of iterations. In each epoch $t$, the SPG/SPP scheme runs for an estimated number of iterations $K_{t}$. More explicitly, the restarted stochastic first order (R-SFO) scheme has the following iteration:

Algorithm 3.1 Let $x_{0,0} \in$ dom F. For $t \geq 1$ compute stepsize $\gamma_{t}$ and number of iterations $K_{t}$. Iterate $S P G / S P P$ algorithm for $K_{t}$ iterations with constant stepsize $\gamma_{t}$ and starting from $x_{K_{t-1}, t-1}$. Set $x_{K_{t}, t}$ the average of iterates.

In the next theorem we derive the convergence rate of this restarted scheme.

Theorem 3.2 Assume that there exist positive constant $B$ and $B_{g}$ such that:

$$
\mathbf{E}\left[\|\nabla f(x, \xi)+\nabla g(x, \xi)\|^{2}\right] \leq B^{2} \text { and }\|\nabla g(x, \xi)\|^{2} \leq B_{g}^{2} \quad \forall x \in \operatorname{dom} F
$$

and that $F$ satisfies a $\nu$ functional growth condition on any segment $[x, \bar{x}]$ :

$$
F(x)-F(\bar{x}) \geq \mu\|x-\bar{x}\|^{\nu} \quad \forall x \in \operatorname{dom} F \quad \text { with } \quad \nu \in[1,2] .
$$

Also let $\gamma_{0}>0, \mathcal{B}^{2}=B^{2}+B_{g}^{2}$ and $\left\{x_{K_{t}, t}\right\}_{t \geq 0}$ be generated by $R$-SFO with stepsize $\gamma_{t}=\frac{\epsilon_{t-1}}{2 \mathcal{B}^{2}}$ and $K_{t}=\left\lceil\frac{4 \mathcal{B}^{2}}{\mu^{2 / \nu} \epsilon_{t-1}^{2-2 / \nu}}\right\rceil$, where $\epsilon_{t}=\frac{\epsilon_{t-1}}{2}$ and $\epsilon_{0} \geq F\left(x_{0,0}\right)-$ $F^{*}$. If, for a given accuracy $\epsilon>0$, we perform $T=\left\lceil\log \left(\frac{\epsilon_{0}}{\epsilon}\right)\right\rceil$ epochs, then after a total number of iterations of the SPG/SPP schemes that is bounded by:

$$
\left\lceil\frac{4 \mathcal{B}^{2}}{\mu^{2 / \nu} \epsilon^{2-2 / \nu}}\right\rceil\left\lceil\log \left(\frac{\epsilon_{0}}{\epsilon}\right)\right\rceil, \quad \text { we have } \quad \mathbb{E}\left[F\left(x_{K_{T}, T}\right)\right]-F^{*} \leq \epsilon .
$$

Proof For any $t \geq 1$, consider the $t$ th epoch of the R-SFO method. Let the sequence $\left\{\epsilon_{t}\right\}_{t \geq 0}$, with $\epsilon_{0} \geq F\left(x_{0,0}\right)-F^{*}$, satisfy:

$$
\mu\left\|x_{K_{t-1}, t-1}-\bar{x}_{K_{t-1}, t-1}\right\|^{\nu} \leq \mathbb{E}\left[F\left(x_{K_{t-1}, t-1}\right)\right]-F^{*} \leq \epsilon_{t-1} .
$$


Also consider the constant stepsize $\gamma_{t}=\frac{\epsilon_{t-1}}{2 \mathcal{B}^{2}}$ and perform $K_{t} \geq \frac{4 \mathcal{B}^{2}}{\mu^{2 / \nu} \epsilon_{t-1}^{2-2 / \nu}}$ number of inner iterations of SPG/SPP. Since (21) holds, then we can use Theorem 2.5 to conclude that:

$$
\begin{aligned}
\mathbb{E}\left[F\left(x_{K_{t}, t}\right)\right]-F^{*} & \leq \frac{\left\|x_{K_{t-1}, t-1}-\bar{x}_{K_{t-1}, t-1}\right\|^{2}}{2 \gamma_{t} K_{t}}+\frac{\gamma_{t} \mathcal{B}^{2}}{2} \\
& \stackrel{(23)}{\leq} \frac{\epsilon_{t-1}^{2 / \nu}}{2 \mu^{2 / \nu} \gamma_{t} K_{t}}+\frac{\gamma_{t} \mathcal{B}^{2}}{2} \leq \frac{\epsilon_{t-1}}{4}+\frac{\epsilon_{t-1}}{4}=\epsilon_{t},
\end{aligned}
$$

which confirms the induction. Since we require $T=\left\lceil\log \left(\epsilon_{0} / \epsilon\right)\right\rceil$ epochs, then we can bound the total number of SPG/SPP iterations as:

$$
\sum_{t=1}^{T} K_{t} \leq \frac{4 \mathcal{B}^{2}}{\mu^{2 / \nu} \epsilon^{2-2 / \nu}} \log \left(\frac{\epsilon_{0}}{\epsilon}\right)
$$

which leads to our statement.

Notice that relation (22) for $\nu=1$ becomes the so-called sharp minima condition [16]. The previous theorem shows that we can still achieve linear convergence for SFO schemes, even when the condition $B=0$ does not hold, provided that the function has a sharp minimum. We only need to implement a restarting variant of these schemes which allows us to obtain linear convergence for the class of functions with bounded subgradients (i.e. $B \neq 0$ ) and having a sharp minima type property (i.e. satisfying the $\nu=1$ functional growth condition (22) ). Moreover, this theorem improves the previous convergence rates of SFO for constant stepsize when $B \neq 0$ and $\nu<2$. More precisely, from Theorem 2.5 we have convergence rate $\mathcal{O}\left(1 / \epsilon^{2}\right)$ for SPP/SPG on the class of functions with $B \neq 0$ and $\nu<2$, while the corresponding restarted variants under a $\nu \in(1,2)$ functional growth condition improves to $\mathcal{O}\left(1 / \epsilon^{2-2 / \nu}\right)$. There are several examples of functions satisfying the sufficient conditions of the previous theorem. It particular, sharp minima condition has been considered frequently in the literature for proving linear convergence of some first order methods, see e.g. [3, 16,21. There are multiple examples of practical optimization models which satisfy (22) with $\nu=1$, in particular objective functions whose epigraph is a polyhedral set: $F(x)=\max _{\xi \in \Omega}\left(a_{\xi}^{T} x+b_{\xi}\right)$. These type of problems arise frequently e.g. in machine learning applications and for discrete stochastic models, that is $f(x)=1 / N \sum_{i=1}^{N} f(x, i)$ and $g(x)=\|x\|_{1}$ or the indicator function of some polyhedral set $C$. We list below the most relevant classes of functions from machine learning applications satisfying (22): hinge loss $f(x, \xi)=\max \left(0,1-y_{\xi} z_{\xi}^{T} x\right)$, absolute loss $f(x, \xi)=\left|z_{\xi}^{T} x-y_{\xi}\right|$ or $\delta$-insensitive loss $f(x, \xi)=\max \left(\left|z_{\xi}^{T} x-y_{\xi}\right|-\delta, 0\right)$. All these instances satisfy also (21) if $g(x, \xi)$ is $\|x\|_{1}$ or the indicator function of some polyhedral set $C_{\xi}$.

Conclusions: In this paper we have derived convergence rates for stochastic first order (SFO) methods with constant or variable stepsize under general assumptions on the optimization problem covering a large class of objective 
functions. In particular, our analysis covered the class of non-smooth Lipschitz functions and composition of a (potentially) non-smooth function and a smooth function, with or without strong convexity. Moreover, given some desired accuracy $\epsilon$, in general, our SFO algorithms are $\epsilon$-free and parametersfree, i.e. the stepsize $\gamma_{t}$ depends on parameters that are easy to compute. E.g., $\gamma_{t}$ depends on $L$ but not on $\mu$, see Theorems 2.6 and 2.7 and also Remark 2.2.

\section{References}

1. Y.F. Atchade, G. Fort and E. Moulines, On stochastic proximal gradient algorithms, https://arxiv.org/abs/1402.2365, 2014.

2. D. Blatt and A.O. Hero, Energy based sensor network source localization via projection onto convex sets, IEEE Transactions on Signal Processing, 54(9): 3614-3619, 2006.

3. J.V. Burke and M.C. Ferris, Weak sharp minima in mathematical programming, SIAM Journal on Control and Optimization, 31(6): 1340-1359, 1993.

4. J. Duchi and Y. Singer, Efficient online and batch learning using forward backward splitting, Journal of Machine Learning Research, 10: 2899-2934, 2009.

5. S. Lacoste-Julien, M. Schmidt and F. Bach, A simpler approach to obtaining an $\mathcal{O}(1 / t)$ convergence rate for projected stochastic subgradient descent, https://arxiv.org/abs/1212.2002, 2012.

6. G. Lan, An optimal method for stochastic composite optimization, Mathematical Programming, 133(1): 365-397, 2012.

7. E. Moulines and F. Bach, Non-asymptotic analysis of stochastic approximation algorithms for machine learning. Advances in Neural Information Processing Systems, 2011.

8. I. Necoara, Yu. Nesterov and F. Glineur, Linear convergence of first order methods for non-strongly convex optimization, Mathematical Programming, 175(1): 69-107, 2019.

9. I. Necoara, P. Richtarik and A. Patrascu, Randomized projection methods for convex feasibility problems: conditioning and convergence rates, Siam Journal on Optimization, 29(4): 2814-2852, 2019

10. I. Necoara, V. Nedelcu and I. Dumitrache, Parallel and distributed optimization methods for estimation and control in networks, Journal of Process Control, 21(5): 756-766, 2011.

11. A. Nedich and D. Bertsekas, Convergence rate of incremental subgradient algorithms, chapter in Stochastic Optimization: Algorithms and Applications, S. Uryasev and P. Pardalos, 263-304, 2000.

12. A. Nemirovski, A. Juditsky, G. Lan and A. Shapiro, Robust stochastic approximation approach to stochastic programming, SIAM Jour. Optimization, 19(4): 1574-1609, 2009.

13. Y. Nesterov, Introductory Lectures on Convex Optimization: A Basic Course, Kluwer, Boston, 2004.

14. A. Patrascu and I. Necoara, Nonasymptotic convergence of stochastic proximal point algorithms for constrained convex optimization, Journal of Machine Learning Research, 18(198): 1-42, 2018.

15. B. Polyak, Minimization of unsmooth functionals, Computational Mathematics and Mathematical Physics, 9(3): 14-29, 1969.

16. B. Polyak, Introduction to optimization, Optimization Software, 1987.

17. L. Rosasco, S. Villa and B.C. Vu, Convergence of stochastic proximal gradient algorithm, Applied Mathematics and Optimization, doi.org/10.1007/s00245-019-09617-7, 2019.

18. E. Ryu and S. Boyd, Stochastic proximal iteration: a non-asymptotic improvement upon stochastic gradient descent, \protect \vrulewidth0pthttp://web.stanford.edu/ eryu/ , 2016.

19. M. Schmidt and N. Le Roux, Fast convergence of stochastic gradient descent under a strong growth condition, https://arxiv.org/abs/1308.6370, 2013.

20. P. Toulis, D. Tran and E.M. Airoldi, Towards stability and optimality in stochastic gradient descent, International Conference on Artificial Intelligence and Statistics, 2016.

21. T. Yang and Q. Lin, RSG: Beating subgradient method without smoothness and strong convexity, Journal of Machine Learning Research, 19(6): 1-33, 2018.

22. L. Xiao and T. Zhang, A proximal stochastic gradient method with progressive variance reduction, SIAM Journal on Optimization, 24(4): 2057-2075, 2015. 